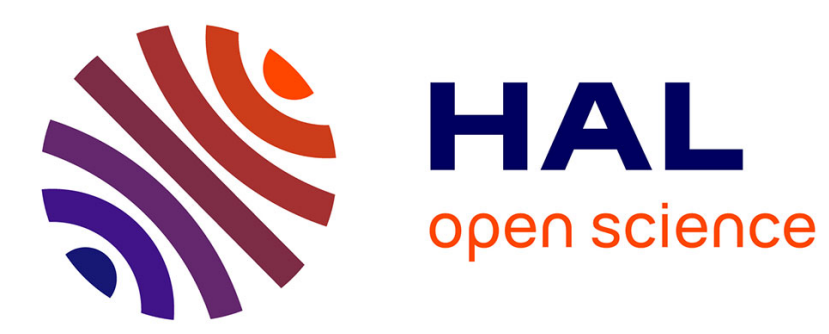

\title{
Direct numerical simulation of turbulent two-phase flows: application to liquid sheet atomization
}

\author{
Stéphane Vincent, Jean-Luc Estivalezes
}

\section{To cite this version:}

Stéphane Vincent, Jean-Luc Estivalezes. Direct numerical simulation of turbulent two-phase flows: application to liquid sheet atomization. CHT-15, May 2015, New Brunswick, France. hal-01172278

\author{
HAL Id: hal-01172278 \\ https://hal.science/hal-01172278
}

Submitted on 9 Jul 2015

HAL is a multi-disciplinary open access archive for the deposit and dissemination of scientific research documents, whether they are published or not. The documents may come from teaching and research institutions in France or abroad, or from public or private research centers.
L'archive ouverte pluridisciplinaire HAL, est destinée au dépôt et à la diffusion de documents scientifiques de niveau recherche, publiés ou non, émanant des établissements d'enseignement et de recherche français ou étrangers, des laboratoires publics ou privés. 
Proceedings of $\mathrm{CHT}-15$

ICHMT International Symposium on Advances in Computational Heat Transfer

May 25-29, 2015, Rutgers University, Piscataway, USA

CHT-15-290

\title{
Direct numerical simulation of turbulent two-phase flows: application to liquid sheet atomization
}

\author{
Stéphane Vincent ${ }^{*}, 0$, Jean-Luc Estivalezes ${ }^{+}$ \\ *Laboratoire de Modélisation et Simulation Multi Echelle, Université Paris-Est, France \\ ${ }^{+}$The French aerospace lab, ONERA-DMAE, Toulouse, France \\ ${ }^{\circ}$ Correspondence author. Email: stephane.vincent@u-pem.fr
}

\begin{abstract}
The atomization of fuel jets in car or plane engines involves complex process with multiphase motions and turbulent flow regimes in a highly coupled way. The Direct Numerical Simulation (DNS) of interfacial flows is investigated in order to characterize the coupling between turbulent flow structures and interface deformations occurring during atomization of a liquid sheet. An incompressible Ghost Fluid formulation of the Navier-Stokes equations is coupled to a level set interface tracking method for representing all the flow and interfacial scales at small scale. A Homogeneous Isotropic Turbulence (HIT) is considered which interacts with an initially flat liquid sheet surrounded by air. The DNS provides detailed simulations used to carry out a parametric study of turbulence-interface interactions according to density and viscosity ratios at the interface as well as surface tension magnitude.
\end{abstract}

\section{INTRODUCTION}

As reported in Trontin et al. [2010], the Direct numerical simulations (DNS) of interface/turbulence interaction are relatively recent (see Lam and Banerjee [1988], Lam and Banerjee [1992]) and generally limited to plane free-surface configurations where the effect of the gas phase on the liquid is neglected and replaced by the free plane surface approximation sometimes in combination with an enforced shear force. Free surface turbulence in an open channel flow was first studied by Lam and Banerjee [1988], Lam and Banerjee [1992]. Lombardi et al. [1996] performed a DNS of countercurrent gas-liquid flow in a channel using free-slip boundary conditions at both channel walls. The interface between the two phases was maintained flat which corresponds to a very high surface tension. It was found that turbulence characteristics on the gas side are similar to those at the near wall and that the lighter phase might be considered as a solid surface at the interface. Handler et al. [1993] conducted similar DNS. In these simulations, the free surface was supposed to be a rigid free-slip wall and the vertical movement of the free surface was neglected. The full free surface boundary conditions were integrated in the work of Komori et al. (1993). But this work was still restricted to small-amplitude surface deformations. A model of wind-generated surface waves was proposed by Borue et al. [1995] by studying the influence of enforced stresses at the free surface. Non-flat interfaces were investigated by De Angelis [1998] by considering stratified flows with a freely deformable interface in the capillary wave regime. Fulgosi et al. [2003] performed DNS of turbulence in a counter-current air-water flow configuration separated by a deformable interface. The results of this work did not differ very much from the previous investigation of Lombardi et al. [1996]. Turbulence intensities, budgets for the Reynolds stresses and flow structures on the gas side showed similarities with results issued from an open channel flow. In Banerjee et al. [2004], friction velocities were considerably increased so as to 
generate surface deformations of higher waveslopes, without leading to wave breaking. It was found by Banerjee et al. [2004] that when the shear rate imposed by the gas is high, turbulence is generated in the vicinity of the interface, like near solid boundaries. In Lakehal et al. [2005], the DNS of the stratified gas-liquid flow over a sheared interface concluded in the necessity to accommodate the asymptotic behaviour of turbulence near interfaces (like in wall flows). The first damping function for near-interface turbulence from the gas side was derived by Lakehal et al. [2005]. In all these studies, the interface deformation is supposed to be moderated excluding strong topological changes such as fragmentation or wave breaking.

More recently, work has been done taking into account fragmentation or break-up in the DNS context of two-phase separated flows while considering interface tracking techniques for handling strong interface deformations. Indeed, Bunner and Tryggvason [2003] have studied the effect of bubble deformation on the properties of turbulent bubbly flows. In their simulations, turbulence was produced by the wake of bubbles. The so-called pseudo-turbulence induced by a bubble swarm was demonstrated not to have the same properties as a well-developed turbulence whose energy spectrum contains an inertial zone. Few DNS studies of deformable bubbles have been carried out in turbulent channel flows. Among them, we can cite Kawamura and Kodama (2002), in which DNS of a fully developed turbulent channel flow containing deformed bubbles are performed. A front-tracking method is used to capture the interface as in Bunner and Tryggvason [2003]. Modifications in the profiles of turbulence intensities were observed. In Liovic and Lakehal [2007a], a LES formulation has been applied to a turbulent bubbling process driven by a constant volume injection of air into a water pool. A strong correlation between turbulence and interface deformations was found. In other works, Toutant et al. [2007] investigated by DNS the motion of a strongly deformable bubble without ruptures in a spatially decaying turbulence in order to perform a priori filtering for LES modelling of interfacial two-phase flows. Liovic and Lakehal [2007b] develop a new strategy around the LES simulation of interfacial flows based on a multiphysics treatment in the vicinity of deformable gas-liquid interfaces. A reconstructed distance function is introduced, on which an interfacial shear velocity is defined to be used in near-interface transport models. This methodology proposed by Liovic and Lakehal [2007b] has been applied for the simulation of a wave breaking configuration.

The aim of the present paper is to investigate DNS of interface/turbulence coupling when a sheet of two immiscible fluids is inserted into a free decaying turbulence. Various density and dynamic viscosity ratios are chosen for constant kinetic viscosities. Different surface tension coefficients are also considered. For various fluid properties, the main goal here is to characterize the turbulence and interface behaviours in terms of interface shape complexity, kinetic energy or probability density functions (PDF) of droplet size.

\section{GHOST FLUID MODEL FOR DNS OF TURBULENT MULTI-PHASE FLOWS}

The ghost fluid model used in Trontin et al. [2010] is implemented in the present work for variable density and viscosity in non-miscible fluids. Incompressible two phase flows are considered. It reads

$$
\begin{aligned}
& \rho\left(\frac{\partial \mathbf{u}}{\partial t}+\mathbf{u} \cdot \nabla \mathbf{u}\right)=-\nabla p+\nabla \cdot\left(\mu\left[\nabla \mathbf{u}+\nabla^{\mathbf{t}} \mathbf{u}\right]\right)+\sigma \kappa \mathbf{n}_{\mathbf{i}} \delta_{\mathbf{i}} \\
& \nabla \cdot \mathbf{u}=\mathbf{0} \\
& \frac{\partial \phi}{\partial t}+\mathbf{u} \cdot \nabla \phi=\mathbf{0}
\end{aligned}
$$

with $\mathbf{u}$ the velocity field, $\mathrm{t}$ the time, $\mathrm{p}$ the pressure, $\rho$ and $\mu$ the density and dynamic viscosity respectively, $\sigma$ the surface tension coefficient, $\kappa$ the local curvature of interface, $\mathbf{n}_{\mathrm{i}}$ the normal to interface and $\delta_{\mathrm{i}}$ the Dirac function indicating interface. The signed distance to the interface, i.e. the level set function $\phi$, is used to provide an implicit representation of the interface location as $\phi=0$. The curvature is obtained as $\kappa=\nabla \cdot\left(\frac{\nabla \phi}{\|\nabla \phi\|}\right)$ whereas $\mathbf{n}_{i} \delta_{i}=\nabla H$ with $\mathrm{H}$ the Heaviside functions defined by 


$$
H=\left\{\begin{array}{cl}
0 & \text { if } \phi<-\frac{3 \Delta x}{2} \\
\frac{1}{2}+\frac{\phi}{3 \Delta x}+\frac{1}{2 \pi} \sin \left(\frac{2 \pi \phi}{3 \Delta x}\right) & \text { if }-\frac{3 \Delta x}{2} \leq \phi \leq \frac{3 \Delta x}{2} \\
1 & \text { if } \phi>\frac{3 \Delta x}{2}
\end{array}\right.
$$

with $\Delta \mathrm{x}$ the local space step on a uniform mesh. The ghost Fluid approach consist in defining the discrete laws that the density and the dynamic viscosity should satisfy in order to verify the physical jump conditions at the interface given by Delhaye [1974]

$$
\begin{aligned}
& {[\mathbf{u}] \cdot \mathbf{n}_{i}=0} \\
& {[\mathbf{u}] \cdot \mathbf{t}_{i}=0} \\
& {\left[-p \mathfrak{I}+\mu\left(\nabla \mathbf{u}+\nabla^{\mathbf{t}} \mathbf{u}\right)\right] \cdot \mathbf{n}_{i}=\boldsymbol{\sigma} \boldsymbol{\kappa} \cdot \mathbf{n}_{i}}
\end{aligned}
$$

where [.] refers to the jump of a quantity at the interface and $\mathfrak{I}$ is the unity tensor of $\mathfrak{R}^{3}$. The local tangent vector to the interface is noted as $\mathbf{t}_{i}$.

The present Ghost fluid model and associated numerical methods (finite volumes, staggered grids, WENO schemes for space discretization of advection/inertial terms, Runge Kuttta TVD schemes for time derivative terms, iterative solvers, and parallel MPI implementation) have been presented and validated into details in Trontin et al. [2008] and Trontin et al. [2010].

\section{INITIAL CONDITIONS FOR HIT FLOW INTERACTING WITH A LIQUID SHEET}

In order to initialize the homogeneous isotropic turbulence (HIT), a computational domain composed of $2 \pi$ cubic box with periodic boundary conditions is considered. Parameters given by Mansour and Wray [1994] are chosen to initiate the HIT field. This field is generated in Fourier space: the constraint of incompressibility is satisfied and the field follows a prescribed energy spectrum. A complete description of the method to initiate an HIT field can be found in Rogallo [1981]. The energy density spectrum of the initial velocity field is given in figure 1 left. It can be observed that with the present DNS code, a $512^{3}$ mesh is required to perform a Reynolds number comparable to a spectral code on $128^{3}$ grid. In the present work, the HIT flow is decaying.

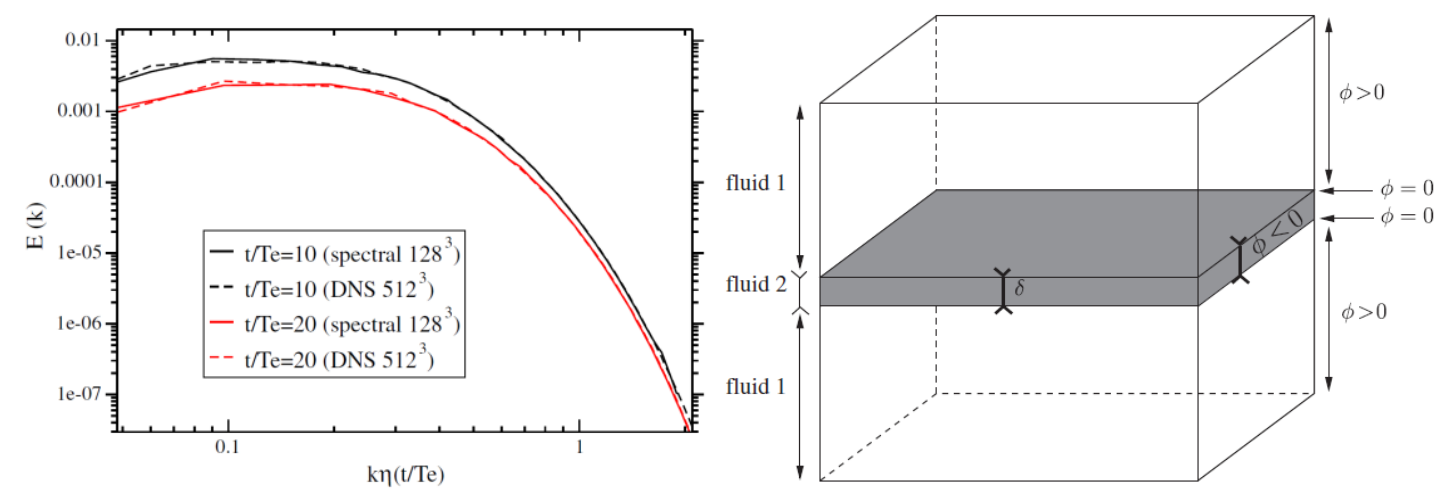

Figure 1. Initial energy density spectrum of the HIT field (left) and initial position of the liquid sheet (fluid 2, right).

A liquid sheet of fluid 2 is initially located at the centre of the periodic box inside the HIT field (figure 1 right). The densities and viscosities are chosen such that the turbulent Reynolds number based on the Kolmogorov scale, and so the kinematic viscosity, is constant and equal to 74 . The initial liquid sheet is flat and its thickness $\delta$ is $5 \%$ of the box size. 


\section{SIMULATION RESULTS FOR TURBULENCE-INTERFACE INTERACTION}

All the simulations have been investigated on a $512^{3}$ grid, which holds for a DNS of the turbulent flows in the present problem. The computational effort was corresponding to using 512 processors during 7 CPU days on the massively parallel computers of the French national facilities GENCI. As was previously observed by Trontin et al. [2010], the Weber number controls the atomization rate of
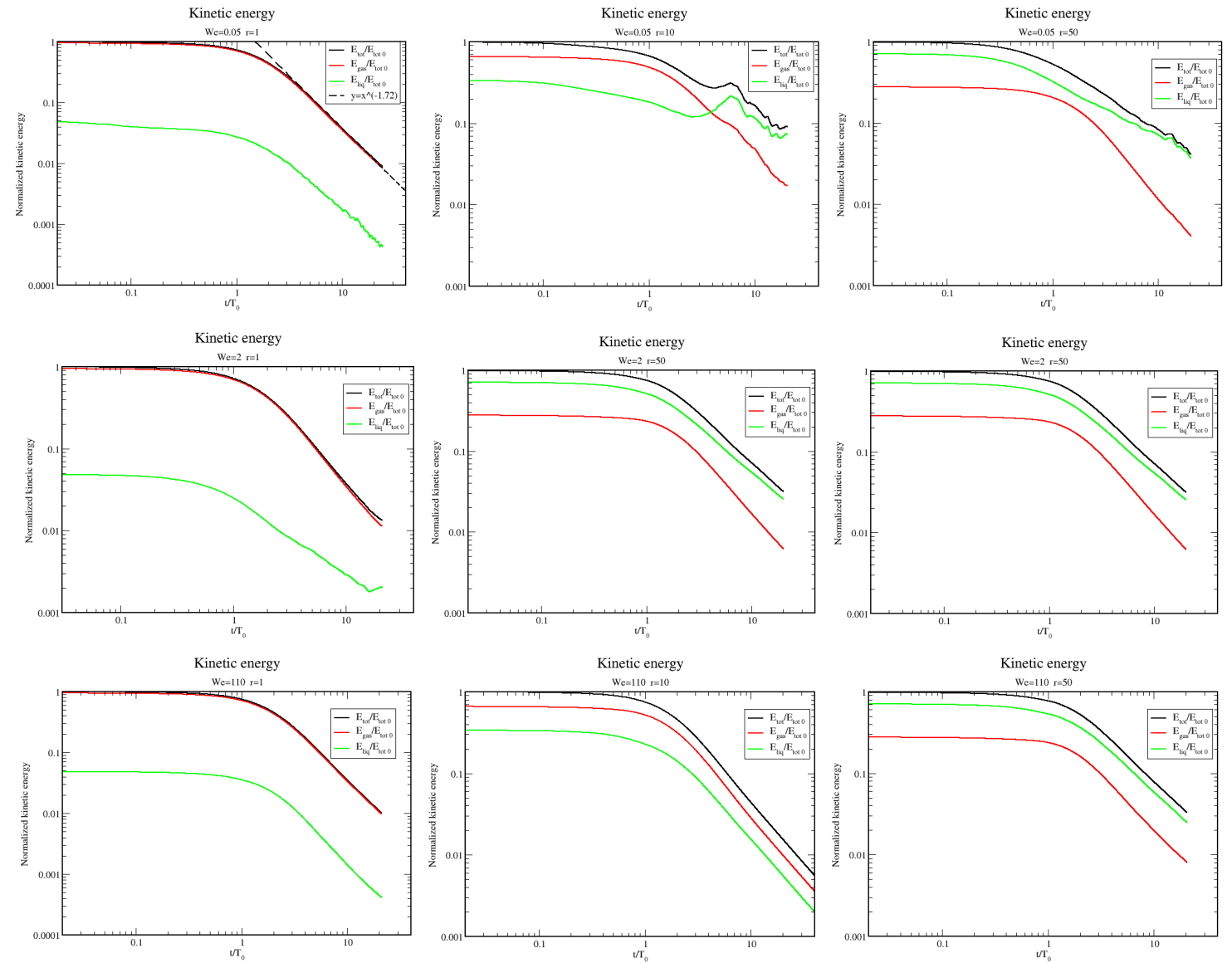

Figure 2. Time evolution of kinetic energy of the HIT field with liquid sheet for $\mathrm{We}=0.05$ (top line), 2 (middle line) and 110 (bottom line). First column stands for $\rho_{2} / \rho_{1}=1$, middle column for $\rho_{2} / \rho_{1}=10$ and right column for $\rho_{2} / \rho_{1}=50$. The black curve is for the total kinetic energy, the red one for the gas and the green one for the liquid.

liquid sheet. For $\mathrm{We}=0.05$, the sheet remains almost planar for $\rho_{2} / \rho_{1}=1$ while for any density ratio, at $\mathrm{We}=2$, big liquid blobs are generated after sheet rupture and at $\mathrm{We}=110$, a spray of liquid drops and ligaments is observed. 
The time evolution of the kinetic energy is first presented in figure 2 for all We numbers and density ratios. It can be observed that whatever the Weber number, the kinetic energy in the liquid phase (the fluid 2) is one order of magnitude less than in the gas, as no added mass nor specific two-phase inertial effects are generated in this case at the interface. The gas occupies $95 \%$ of the volume and its kinetic energy dominates as expected. For density ratio of 10, the liquid kinetic energy can be larger $(\mathrm{We}=2)$ or smaller $(\mathrm{We}=110)$ and also both during time $(\mathrm{We}=0.05)$ depending on the energy transfers at the interface. In this case, the gas kinetic energy remains important. For density ratio of 50, the kinetic energy in the liquid is dominant for all Weber numbers, corresponding to a fully atomized regime.

The distribution of liquid droplet size (PDF) is reported in figure 3 for $\mathrm{We}=2$ and 110, i.e. when the sheet has broken. Four different times are given, corresponding to 5, 10, 15 and 20 eddy turnover time $\mathrm{T}_{0}$, also called Eulerian time scale. Its value is $0.278 \mathrm{~s}$ in the present simulations. Weber number 0.05 is not presented in terms of PDF as no fragmentation occurs in this case. It can be observed that the larger the density ratio is, the larger is the PDF of the droplet size for $\mathrm{We}=2$ and 110 . The number of classes of droplet sizes increases with inertial effects. These numerical characterizations are of first importance for combustion processes in engines as the droplet size distribution controls the rate of energy exchange and chemical reactions.

Other interesting coupling variables are under investigation in the present work, such as the kinetic energy spectra transformation for various We and density ratios. They will be presented during the oral presentation.

\section{CONCLUSION}


The direct numerical simulation of the fragmentation of a liquid sheet has been investigated when interacting with a decaying homogeneous isotropic turbulence. A ghost fluid/level set approach has been implemented for simulating at the local scale the interface deformations and the turbulence transformation. It has been observed that the time evolution of the kinetic energy in gas and liquid is highly depend on the density ratio at the interface for a constant turbulence Reynolds number of 74 . In the same way, the droplet size distributions (PDF) are strongly affected and enlarged as soon as the density ratio is increased.

\section{REFERENCES}

De Angelis, V. [1998] Numerical Investigation and Modelling of Mass Transfert Processes at Sheared Gas-liquid Interface, $\mathrm{PhD}$ Thesis, UCSB.

Banerjee, S. et al. [2004] Surface divergence models for scalar exchange between turbulent streams. Int. J. Multiphase Flow, Vol. 30, pp 963-977.

Borue, V. et al. [1995] Interaction of surface waves with turbulence: direct numerical simulations of turbulent open-channel flow, J. Fluid Mech., Vol. 286, pp 1-23.
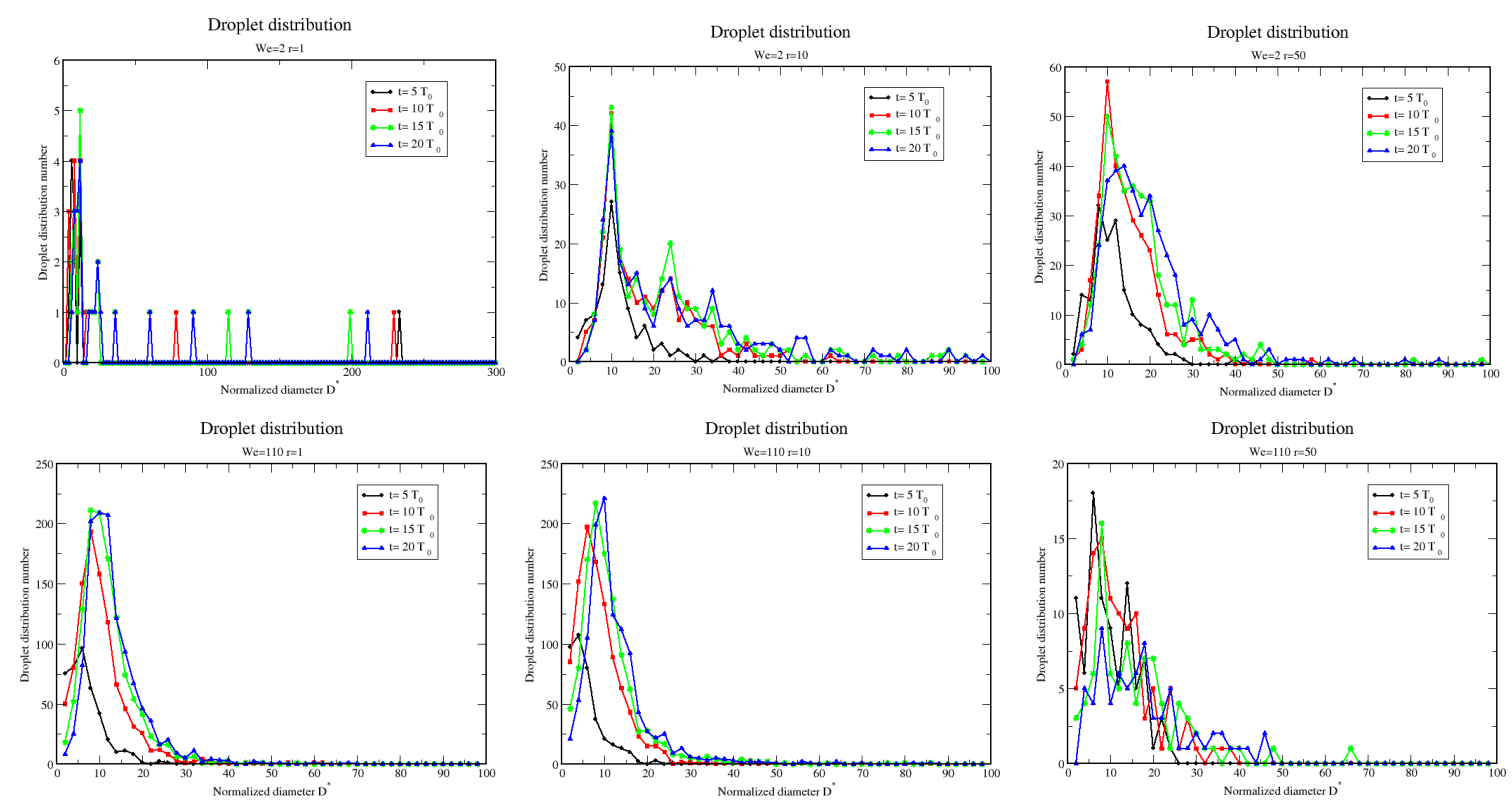

Figure 3. Probability density functions (PDF) of droplet size for $\mathrm{We}=2$ (top line) and $\mathrm{We}=110$ (bottom column). From left to right, $\rho_{2} / \rho_{1}=1,10$ and 50 . Time $5 \mathrm{~T}_{0}$ (black line), 10 $\mathrm{T}_{0}$ (red line), $15 \mathrm{~T}_{0}$ (green line) and $20 \mathrm{~T}_{0}$ (blue line) are plotted.

Bunner, B. and Tryggvason, G. [2003], Effect of bubble deformation on the properties of bubbly flows, J. Fluid Mech., Vol. 495, pp 77-118.

Delhaye, J. [1974] Jump conditions and entropy sources in two-phase systems. Local instant formulation. Int. J. Multiphase Flow, Vol. 1, pp 395-409.

Fulgosi, M. et al. [2003] Direct numerical simulation of turbulence in a sheared air-water flow with deformable interface, J. Fluid. Mech, Vol. 482, pp 319-345.

Handler, R.A. et al. [1993] Length scales and the energy balance for turbulence near a free surface, AIAA J., Vol. 31 (11), pp 1998-2007.

Kawamura, T. and Kodama, Y. [2002] Numerical simulation method to resolve interactions between bubbles and turbulence, Int. J. Heat Fluid Flow, Vol. 23, pp 627-638.

Lakehal, D. et al. [2005] SGS modelling for the LES of interfacial gas-liquid flows, La Houille Blanche, Rev. Int. L'eau, Vol. 6, pp 125-131. 
Lam, K. and Banerjee, S. [1988] Investigation of turbulent flow bounded by a wall and a free surface, In: Michaelides, E.E., Sharma, M.P. (Eds.), Fundamentals of Gas-Liquid Flows, vol. 72. Springer, New York, pp 29-38.

Lam, K. and Banerjee, S. [1992] On the condition of streaks formation in a bounded turbulent flow, Phys. Fluids, Vol. A4, pp 306-320.

Liovic, P. and Lakehal, D. [2007a] Interface-turbulence interactions in large-scale bubbling processes. Int. J. Heat Fluid Flow, Vol. 28, pp 127-144.

Liovic, P. and Lakehal, D. [2007b] Multi-physics treatment in the vicinity of arbitrarily deformable gas-liquid interfaces, J. Comput. Phys., Vol. 222, pp 504-535.

Lombardi, P. et al. [1996] Direct numerical simulation of near interface turbulence in coupled gasliquid flow. Phys. Fluids, Vol. 8, pp 1643-1665.

Mansour, N.N. and Wray, A.A. [1994] Decay of isotropic turbulence at low Reynolds number, Phys. Fluids, Vol. 6 (2), pp 808-814.

Rogallo, R.S. [1981] Numerical Experiments in Homogeneous Turbulence. Technical Report TM 81315, NASA.

Toutant, A. et al. [2007] Interfacial conditions at a filtered interface for les two-phase flows, In: ICMF 2007, Germany.

Trontin, P. et al. [2008] Detailed comparisons of front-capturing methods for turbulent two-phase flows simulations. Int. J. Numer. Methods Fluids, Vol. 56 (8), pp 1543-1549.

Trontin, P. et al. [2010] Direct numerical simulation of a freely decaying turbulent interfacial flow, Int. J. Multiphase Flow, Vol. 36, pp 891-907. 